\title{
A saúde e o envelhecimento de pessoas com Síndrome de Down: O que dizem os
}

\section{trabalhadores da Atenção Primária à Saúde}

\author{
Health and aging of people with Down Syndrome: What workers in primary health care say \\ Salud y envejecimiento de personas con Síndrome de Down: Lo que dicen los trabajadores de \\ atención primaria de salud
}

Recebido: 05/07/2021 | Revisado: 11/07/2021 | Aceito: 14/07/2021 | Publicado: 24/07/2021

\author{
Rosane Seeger da Silva \\ ORCID: https://orcid.org/0000-0001-7980-4762 \\ Universidade Federal de Santa Maria, Brasil \\ E-mail: rosane.seeger@hotmail.com.br \\ Fernanda dos Santos Pascotini \\ ORCID: https://orcid.org/0000-0001-6244-2795 \\ Universidade Federal de Santa Maria, Brasil \\ E-mail: fepascotini@hotmail.com \\ Elenir Fedosse \\ ORCID: https://orcid.org/0000-0002-9691-7491 \\ Universidade Federal de Santa Maria, Brasil \\ E-mail: efedosse@gmail.com
}

\begin{abstract}
Resumo
Analisar a compreensão de trabalhadores da Atenção Primária à Saúde sobre a saúde e a condição de envelhecimento de pessoas com Síndrome de Down. Abordagem quali-quantitativa, exploratório, realizado com trabalhadores da Atenção Primária à Saúde de um município do sul do Brasil. Os dados foram coletados por meio de entrevistas, realizando-se análise do Discurso do Sujeito Coletivo para os dados qualitativos e os quantitativos, tratados descritivamente. Participaram 57 trabalhadores. Houve predominância do sexo feminino (82,5\%). A média geral de idade, independentemente do sexo, foi de 44,8 anos. A partir da análise do Discurso do Sujeito Coletivo, emergiram quatro temas: i) concepções dos trabalhadores sobre pessoas com Síndrome de Down; ii) a percepção de trabalhadores sobre a saúde de pessoas com Síndrome de Down; iii) o cuidado integral de pessoas com Síndrome de Down e iv) o (re)conhecimento da Avaliação Multidimensional Rápida da Pessoa Idosa e a prospecção do cuidado em saúde de pessoas com Síndrome de Down. Constatou-se que o cuidado de pessoas com Síndrome de Down ainda está fortemente vinculado aos serviços especializados, ou seja, é frágil a relação entre essas pessoas e a Atenção Primária à Saúde, está atrelada além das diferenças físicas, as cognitivas e de comportamento dessas pessoas.
\end{abstract}

Palavras-chave: Saúde; Envelhecimento; Síndrome de Down; Trabalhador da saúde; Atenção primária à saúde.

\begin{abstract}
To analyze the understanding of Primary Health Care workers about the health and aging condition of people with Down Syndrome. Qualitative-quantitative, exploratory approach, carried out with Primary Health Care workers in a municipality in southern Brazil. The data were collected through interviews, analyzing the Collective Subject Discourse for qualitative and quantitative data, which were treated descriptively. 57 workers participated. There was a predominance of females $(82.5 \%)$. The general average age, regardless of gender, was 44.8 years. From the analysis of the Collective Subject Discourse, four themes emerged: i) workers' conceptions about people with Down Syndrome; ii) the perception of workers about the health of people with Down Syndrome; iii) comprehensive care for people with Down Syndrome and iv) (re) knowledge of the Multidimensional Rapid Assessment of the Elderly and the prospecting of health care for people with Down Syndrome. It was found that the care of people with Down Syndrome is still strongly linked to specialized services, that is, the relationship between these people and Primary Health Care is fragile, it is linked beyond their physical, cognitive and behavioral differences people.
\end{abstract}

Keywords: Health; Aging; Down syndrome; Health worker; Primary health care.

\section{Resumen}

Analizar la comprensión de los trabajadores de Atención Primaria de Salud sobre la salud y el envejecimiento de las personas con Síndrome de Down. Abordaje cualitativo-cuantitativo, exploratorio, realizado con trabajadores de Atención Primaria de Salud en un municipio del sur de Brasil. Los datos fueron recolectados a través de entrevistas, analizando el Discurso Colectivo del Sujeto en busca de datos cualitativos y cuantitativos, tratados de manera descriptiva. Participaron 57 trabajadores. Predominó el sexo femenino (82,5\%). La edad media general, independientemente del sexo, fue de 44,8 años. Del análisis del Discurso del Sujeto Colectivo surgieron cuatro temas: 
i) las concepciones de los trabajadores sobre las personas con Síndrome de Down; ii) la percepción de los trabajadores sobre la salud de las personas con síndrome de Down; iii) atención integral a personas con Síndrome de Down y iv) (re) conocimiento de la Evaluación Rápida Multidimensional del Anciano y la prospección de la atención de salud a personas con Síndrome de Down. Se encontró que la atención a las personas con Síndrome de Down sigue estando fuertemente ligada a los servicios especializados, es decir, la relación entre estas personas y la Atención Primaria de Salud es frágil, está ligada más allá de sus diferencias físicas, cognitivas y conductuales personas.

Palabras clave: Salud; Envejecimiento; Síndrome de Down; Trabajador de la salud; Primeros auxilios.

\section{Introdução}

A Síndrome de Down (SD) é um distúrbio genético caracterizado pela trissomia do cromossomo no par 21. Apresenta características físicas específicas (formato diferenciado/achatamento da cabeça; boca permanentemente entreaberta - por hipotonia do órgão do sistema estomatognático; pregas epicântricas; orelhas pequenas; palma da mão com uma linha cruzada [linha simiesca]; distância entre dedos dos pés aumentada, entre outros), comprometimentos no desenvolvimento neuropsicomotor, acompanhado de deficiência intelectual (Pueschel, 2003; Rachidi \& Lopes, 2008; Brasil, 2013). O diagnóstico clínico já pode ser realizado ao nascimento, pelas aparentes características físicas, sendo confirmada por análise cromossômica (Ribeiro et al., 2003).

Devido aos avanços tecnológicos e da medicina a expectativa de vida de uma pessoa com SD tem aumentado nos últimos anos, sendo esperada sobrevida acima de 50 anos; uma a cada 10 alcançando os 70 anos de idade (Wiseman et al., 2015). Com o aumento da expectativa de vida, essas pessoas podem se deparar com situações de adoecimento como, por exemplo, as condições crônicas de saúde (Hipertensão Arterial Sistêmica [HAS], Diabetes Mellitus [DM], entre outras), que possuem características de início e evolução lentos. Tais condições apresentam como principais fatores de risco o sedentarismo, a alimentação inadequada, a hereditariedade, a exposição a fatores ambientais e, também o envelhecimento (Brasil, 2006; Mendes, 2012).

Caracterizam-se como condição crônica de saúde as, anteriormente, chamadas doenças crônicas não transmissíveis (DCNT), as doenças infecciosas persistentes, os distúrbios mentais, a deficiência física e as condições ligadas à manutenção da saúde por ciclos de vida (Mendes, 2012). As condições crônicas representam um alto ônus, tanto para as pessoas que as têm e seus familiares quanto para o sistema de saúde, em função dos custos com cuidados e sobrecarga medicamentosa (Valcarenghi et al., 2015). Por isso, entende-se a necessidade de ações contínuas, proativas e integradas, do sistema de atenção à saúde, de seus trabalhadores e das pessoas usuárias para o controle efetivo, eficiente e com qualidade das condições crônicas de saúde (Mendes, 2012; Silva et al., 2017).

Reconhece-se que a assistência a pessoas com SD deve envolver uma equipe multiprofissional, cujo atendimento deve ser fundamentado em diretrizes clínicas, baseado em evidências de informações relevantes e ações organizadas para que recebam a atenção adequada às suas necessidades. As ações podem ser individuais ou em grupos e incluem a prevenção de doenças e agravos, bem como o estímulo ao autocuidado (Mendes, 2012; Goulart, 2012). Estudos indicam, ainda, que a atenção à saúde de pessoas com SD deve pautar-se em um modelo de cuidado com foco nos direitos, necessidades, preferências e habilidades desses usuários, sendo a assistência multiprofissional humanizada - criação de vínculos pautados na ética, compromisso e respeito. Contudo, também revelam a necessidade de mecanismos de sensibilização e ação social quantos aos aspectos relacionados à prevenção e à promoção de saúde para um envelhecer saudável (Brasil, 2006; Vello et al., 2014; Silva et al., 2017).

A Atenção Primária à Saúde (APS) configura-se como a principal porta de entrada para o Sistema Único de Saúde (SUS); espera-se que aproximadamente $80 \%$ das necessidades de atenção à saúde de uma comunidade adstrita devam ser resolvidas neste nível de atenção à saúde (Mendes, 2012; Duncan, 2012). Sendo assim, os serviços de APS, especialmente, as 
unidades de Estratégia de Saúde da Família (ESF) são locus determinante para ampliação da assistência e da visão acerca do processo saúde-doença de todo e qualquer usuário (Brasil, 2006), inclusive de pessoas com SD.

A partir do dito anteriormente, perguntou-se: pessoas com SD que requerem acompanhamento contínuo por parte da saúde e da educação, têm sido atendidas na APS? Aspectos relacionados ao envelhecimento têm sido contemplados pelos trabalhadores da APS? Logo, o objetivo do presente estudo foi analisar a compreensão de trabalhadores da APS sobre a saúde e a condição de envelhecimento de pessoas com SD.

\section{Metodologia}

Trata-se de um estudo exploratório e de abordagem quali-quantitativa, realizado junto à trabalhadores da APS (identificados quanto ao sexo, idade, ocupação/profissão, grau e tempo de formação, bem como tempo de atuação no serviço) de um município de médio porte do interior do estado do Rio Grande do Sul/RS. Para viabilizá-la foram realizadas entrevistas, com questões norteadoras (Quadro 1), elaboradas exclusivamente para este estudo, visando identificar a concepção dos trabalhadores da APS quanto às pessoas com SD, suas condições e necessidades de saúde, bem como verificar o conhecimento e/ou atuação dos trabalhadores acerca do processo de envelhecimento e das formas de avaliação de pessoas com SD envelhecentes. Destaca-se que, quando um trabalhador referia não ter conhecimento acerca de como avaliar e acompanhar uma pessoa idosa, a pesquisadora apresentava brevemente a Avaliação Multidimensional Rápida da Pessoa Idosa (AMRPI) que integra o Caderno de Atenção Básica - nº 19, do Ministério da Saúde (Brasil, 2006).

Quadro 1 - Questões norteadoras da elaboração dos Discursos dos Sujeitos Coletivos.

\begin{tabular}{|l|}
\hline Tema I - As concepções de trabalhadores da APS sobre as pessoas com SD \\
\hline 1) Durante a sua formação profissional foram abordados conhecimentos sobre SD? \\
\hline 2) Durante a graduação teve contato com pessoas com SD? \\
\hline Tema II: As percepções de trabalhadores da APS sobre a saúde de pessoas com SD \\
\hline 1) Atualmente, você atende pessoas com SD? \\
\hline 2) Como você considera essa experiência? \\
\hline 3) Como você caracteriza a saúde de pessoas com SD? \\
\hline Tema III: O cuidado integral de pessoas com SD \\
\hline 1) O que você conhece sobre o processo de envelhecimento de pessoas com SD? \\
\hline 2) Como você avalia o cuidado prestado pela sua equipe a pessoas com SD? \\
\hline Tema IV: O (re)conhecimento da AMRPI e a prospecção do cuidado em saúde de pessoas com \\
SD \\
\hline 1) Você conhece e/ou usa a AMRPI? \\
\hline 2) Você considera pertinente avaliar a saúde de pessoas com SD envelhecentes sob a perspectiva da \\
AMRPI?
\end{tabular}

Fonte: Autores (2020).

A amostra foi por conveniência (Minayo, 2014), tendo como participantes: Agentes Comunitários de Saúde (ACS), Auxiliares de Consultório Dentário (ACD), cirurgiões dentistas, enfermeiros, médicos e técnicos de enfermagem, os quais integram as equipes mínimas da APS. Também participaram uma fisioterapeuta, uma fonoaudióloga e uma nutricionista (residentes do segundo ano de um Programa de Residência Multiprofissional na APS). Os profissionais foram convidados a participarem mediante convite realizado nas reuniões de equipe (que ocorrem semanalmente em todas as unidades de saúde), ou seja, em seus locais de trabalho. Nesta ocasião foram agendadas as entrevistas. 
Quando entrevistados, afirmaram sua participação por meio da assinatura do Termo de Consentimento Livre e Esclarecido (TCLE), em duas vias (uma que ficou em posse do participante e a outra com a pesquisadora). Os critérios de inclusão adotados foram: ser trabalhador atuante na APS há mais de seis meses. Adotou-se como critério de exclusão: trabalhador recém-contratado, afastado ou em férias no dia da coleta agendada.

As entrevistas foram gravadas em áudio, com duração de 15 a 20 minutos cada, tendo sido garantidos o sigilo das informações e o anonimato dos participantes. Cada participante foi identificado com a letra "T" (Trabalhador), seguida de um número correspondente à ordem das entrevistas (T1; T2; T3; ...). Ressalta-se, portanto, que esta pesquisa cumpriu as recomendações da Resolução no 466/12, do Conselho Nacional de Saúde (Brasil, 2012) e foi aprovada pelo Comitê de Ética em Pesquisa da Universidade onde ela ocorreu, sob Parecer n 2.810 .565 (emitido em 9 de agosto de 2018).

\section{Resultados e Discussão}

Participaram 57 trabalhadores, conforme demonstrado na Tabela 1. Os dados, apresentados em ordem decrescente, referem-se às características sociodemográficas, experiência profissional e sondagem quanto à assistência prestada às pessoas com SD na APS.

Tabela 1 - Perfil de trabalhadores da Atenção Primária à Saúde participantes do estudo (n=57).

\begin{tabular}{|c|c|c|c|}
\hline \multicolumn{2}{|c|}{ VARIÁVEL } & $\mathbf{N}$ & $\%$ \\
\hline \multirow{2}{*}{ Sexo } & Feminino & 47 & 82,5 \\
\hline & Masculino & 10 & 17,5 \\
\hline \multirow{9}{*}{ Profissão } & ACS & 26 & 45,6 \\
\hline & Enfermeiro & 16 & 28,1 \\
\hline & Médico & 6 & 10,5 \\
\hline & Cirurgiões Dentista & 3 & 5,3 \\
\hline & $\begin{array}{c}\text { Técnico } \\
\text { Enfermagem }\end{array}$ & 2 & 3,5 \\
\hline & ACD & 1 & 1,7 \\
\hline & Fisioterapeuta & 1 & 1,7 \\
\hline & Fonoaudiólogo & 1 & 1,7 \\
\hline & Nutricionista & 1 & 1,7 \\
\hline TOTAL & & 57 & 100 \\
\hline \multirow{2}{*}{$\begin{array}{l}\text { Assistência a pessoas com } \\
\text { Síndrome de Down }\end{array}$} & Sim & 56 & 98,2 \\
\hline & Não & 1 & 1,8 \\
\hline
\end{tabular}

Legenda: ACS = Agente Comunitário de Saúde; ACD = Auxiliar de Consultório Dentário; APS =Atenção Primária à Saúde.

Fonte: Dados da pesquisa (2019).

Note-se que o predomínio dos sujeitos da pesquisa foi de mulheres: destacam-se as profissões de ACS (88,5\% eram mulheres), Enfermagem, Odontologia, Fisioterapia e Fonoaudiologia. A feminização da força de trabalho na APS é considerada uma tendência, em especial nas unidades de Saúde da Família que contam com ACS (Marsiglia, 2011). Os dados deste estudo podem ser explicados pela construção histórica e cultural do papel da mulher na sociedade, sobretudo a noção de cuidado em saúde percebida como ação feminina (Pastore, Rosa \& Homem, 2008; Wermelinger et al., 2010).

A média geral de idade dos participantes, independentemente do sexo, foi de 44,8 anos (DP \pm 9,95 anos). Tal perfil corrobora outros estudos na área da saúde (Canesqui \& Spinelli, 2006; Giordani et al. 2015). Quanto ao tempo na profissão e de atuação na APS, obtiveram-se desvios padrões de 8,93 anos e de 5,97 anos, respectivamente. Resultados similares ou 
correspondentes a tais informações não foram encontrados na literatura, mas podem ser interpretados como decorrentes do fato de o Programa Saúde da Família ter sido implantado em 2006, pela Portaria no 648/2006 (Brasil, 2006b), emergindo oportunidades para inclusão de mais e novos núcleos profissionais da saúde na perspectiva de ampliação do cuidado integral.

A Tabela 2, apresenta a especificação da formação profissional dos trabalhadores da APS participantes deste estudo.

Tabela 2 - Especificação da formação dos trabalhadores da Atenção Primária à Saúde (n= 57).

\begin{tabular}{|c|c|c|}
\hline CARGO & FORMAÇÃO & $\mathbf{N}$ \\
\hline \multirow{4}{*}{ Agente Comunitário de Saúde } & Nível Médio Técnico & 14 \\
\hline & Nível Médio (Ensino Médio) & 5 \\
\hline & Nível Superior Completo & 4 \\
\hline & Nível Superior Incompleto & 3 \\
\hline \multirow{5}{*}{ Enfermeiro } & Especialista em Saúde Coletiva & 2 \\
\hline & $\begin{array}{l}\text { Especialista em Saúde da } \\
\text { Família }\end{array}$ & 6 \\
\hline & Mestrado em Enfermagem & 3 \\
\hline & $\begin{array}{l}\text { Especialista em Saúde do } \\
\text { Trabalhador }\end{array}$ & 1 \\
\hline & Nível Superior & 4 \\
\hline Médico & $\begin{array}{llll}\text { Especialista em } & \text { Saúde } & \text { da } \\
\text { Família } & & & \end{array}$ & 6 \\
\hline \multirow{3}{*}{ Dentista } & Nível Superior & 1 \\
\hline & Especialista em Odontopediatria & 1 \\
\hline & Especialista em Saúde Coletiva & 1 \\
\hline Técnico em Enfermagem & Nível Médio Técnico & 2 \\
\hline Auxiliar de Consultório Dentário & Nível Médio Técnico & 1 \\
\hline Fisioterapeuta & $\begin{array}{l}\text { Especialização Incompleta na } \\
\text { área }\end{array}$ & 1 \\
\hline Fonoaudiólogo & $\begin{array}{l}\text { Especialização Incompleta na } \\
\text { área }\end{array}$ & 1 \\
\hline Nutricionista & $\begin{array}{l}\text { Especialização Incompleta na } \\
\text { área }\end{array}$ & 1 \\
\hline TOTAL & & 57 \\
\hline
\end{tabular}

Fonte: Dados da pesquisa (2019).

Conforme a Tabela 2, verifica-se que a formação da população estudada é qualificada, um dado importante na medida em que a APS exige profissionais cada vez mais preparados e qualificados para melhor desempenho de suas atividades laborais (Ribeiro \& Martins, 2011). Constatou-se que, aproximadamente, a metade dos ACS possuía nível de instrução técnica (34,6\% eram técnicos em enfermagem; $3,8 \%$ técnicos em segurança do trabalho; igual porcentagem de técnicos em radiologia, em secretariado e em contabilidade), enquanto a outra metade cursou ou cursava nível superior (3,8\% eram assistentes sociais; igual porcentagem de arquivologista, pedagogo, fonoaudiólogo e geógrafo). Tal situação pode facilitar o processo de trabalho nas ESF, visto que eles tendem a atuar com ampliada concepção de saúde e, como eles são importantes elos entre a comunidade e os serviços (Resende et al., 2011), podem melhor analisar as condições e as necessidades de saúde dos usuários que acompanham.

Destaca-se a participação de fisioterapeuta, fonoaudiólogo e nutricionista neste estudo, ressaltando-se que são residentes de um programa de Residência Multiprofissional em Saúde com ênfase na APS. Pode-se afirmar que essa situação não é comum na APS, visto que a inserção dos referidos profissionais, tal como de outros (assistentes sociais, psicólogos, 
terapeutas ocupacionais, entre outros), tende a ser por meio das Equipes do Núcleo Ampliado de Saúde da Família e Atenção Primária (NASF-AP) (Brasil, 2012). Os discursos de tais profissionais se destacaram no que se refere aos conceitos de interdisciplinaridade e/ou comunicação entre os profissionais, integralidade do cuidado e inclusão social.

\section{Discursos dos Sujeitos Coletivos}

Apresentam-se, aqui, os DSC organizados em quatro grandes temas: - i) as concepções de trabalhadores da APS sobre as pessoas com SD; ii) as percepções dos trabalhadores da APS sobre a saúde de pessoas com SD; iii) o cuidado integral de pessoas com SD; iv) o (re)conhecimento da AMRPI e a prospecção do cuidado em saúde de pessoas com SD.

\section{Tema I: As concepções de trabalhadores da Atenção Primária à Saúde sobre as pessoas com Síndrome de Down}

O DSC 1 representa as concepções de trabalhadores da saúde com formação em nível médio e técnico que apresentaram contato regular, apesar de não cotidiano, com pessoas com SD.

DSC 1: Eu acho que ela [a pessoa com SD] vai ter grande possibilidade de ter uma vida normal - estudar, trabalhar, se relacionar .... Agora a gente vê que existem pessoas com SD se desenvolvendo normal. Eles são normais. Levam uma vida normal. É um componente da família normal, como os demais filhos. Mas... quando eu era jovem, eu conheci, nas famílias, pessoas com essa SD que eram isoladas. A gente era curioso e ficava se perguntando: "por que que não tratavam como os demais?" "Por que eles não eram tratados como nós crianças assim, né?" "Por que eles não frequentavam a escola?" Não tinha escola para eles. Hoje, eles são considerados pessoas normais como a gente, um pouquinho diferente o rosto, o olho, enfim são as características físicas que mudam.

Note-se que este DSC condiz com a noção de pessoas com SD como "normais", parecendo indicar que as diferenças físicas são quase irrelevantes. A propósito, a decisão sobre o que fazer com o outro, o diferente, percorre os séculos. Da Antiguidade à Idade Média, a pessoa com deficiência era vista como um subumano ou concebida como sobrenatural, digna de atitudes antagônicas extremas; por isso as atitudes dispensadas a eles eram de negligências e maus tratos (Aranha, 2001).

O DSC 2 corresponde às concepções de trabalhadores, também nível médio técnico, porém distinta da anterior colocando as pessoas com SD como "diferentes" destacando as diferenças físicas, cognitivas e de comportamento dessas pessoas. Revelou, ainda, uma visão de que as vulnerabilidades sociais e o preconceito desfavorecem o desenvolvimento e aceitação dessas pessoas.

DSC 2: É diferente de um ser humano normal, tem várias coisas diferentes no organismo dele. É um ser humano que é mutante. Por isso, funciona de maneira diferente. Alguns tem um pouco mais de deficiência intelectual e infelizmente são mais debilitados pela questão da vulnerabilidade social... Isso aí é bem difícil. Eu acho que eles têm potencial, porém os estímulos são diferentes. Ainda estão rotulando muito como uma pessoa doente, uma pessoa incapaz, né? Não estão tratando como uma pessoa que pode conviver em sociedade normalmente como nós.... É uma criança grande. São bem comunicativos, carinhosos, amorosos, bem tranquilos. Eu acho que estão sendo tratados muito assim: com diferença. Eles lá e a gente aqui; o mundo dela e o mundo da gente, com diferença!

O DSC 2 parece retratar uma visão mais realista, ou seja, mais em conformidade com a condição reconhecida por grande parte dos profissionais e pela sociedade em geral. Neste sentido, Werneck (2006) destaca que as formas sutis de discriminação, mesmo com o propósito de valorizar as pessoas com deficiência, acabam segregando-as cada vez mais. O simples fato de considerá-las especiais já as distâncias do gozo incondicional dos Direitos Humanos, gozo que antecede qualquer norma nacional ou internacional (Werneck, 2006).

O DSC 3 traz as concepções de trabalhadores da saúde com formação superior e com contato restrito com pessoas com SD, similar a dos trabalhadores que geraram o DSC 1 . 
DSC 3: Eu considero normal, natural. A gente sabe que o Down tem uma variação muito grande né, pode ter uma deficiência mental, mas a inteligência pode ser normal. Tem Down formado em Direito, em Fisioterapia, em várias áreas aí e exercendo uma profissão. Então.... Eu acho que tem que investir nessa capacidade deles que a gente sabe que eles têm, com um pouquinho mais de esforço. A cultura da família interfere também, né? Ela [pessoa com SD] pode ser bem desenvolvida.

Note-se que o DSC acima evidencia as concepções de que são possíveis e bem-sucedidas as correções dos problemas orgânicos (Huber et al., 2010), bem como evidenciam as potencialidades do desenvolvimento desde que acompanhados por especialistas. Estudos revelam que a estimulação cognitiva e educacional desde tenra idade (estimulação precoce) pode trazer benefícios as pessoas com SD (Giaccini, Tonial \& Mota, 2013; Freire, 2014). Segundo Mattos \& Bellane (2010) a intervenção é considerada benéfica, quando iniciada precocemente antes que os padrões de postura e movimentos atípicos tenham sido já instalados, ou seja, nos primeiros quatro meses de vida do bebê seria a época essencial para se iniciar o programa de intervenção.

O DSC 4, também retrata as concepções de trabalhadores de saúde de nível superior, tal qual como o DSC 2, destaca a condição de que as pessoas com SD serem "pessoas diferentes". No entanto, ainda se evidencia que a diferença das pessoas com SD pode ser exacerbada com a falta de conhecimentos e habilidades profissionais. As representações, ideias, imagens e conceitos sobre a SD e as deficiências em geral, mudaram através dos tempos, foram e voltaram e algumas permanecem (Casarin, 2001).

DSC 4: Eu atendo como se fosse um paciente normal. Mas, eu me dedico mais, tem que ter mais paciência...porque eles são mais inquietos. Então... eu vejo assim: tu tens que tratar como normal e só ter um pouquinho mais de paciência. São pessoas ainda excluídas. Eu os vejo como excluídos. São incluídos no reduto delas né? Mas na sociedade em geral, eu vejo uma exclusão ainda. Talvez até porque as pessoas não saibam trabalhar com eles; com essa diferença, talvez seja isso também. É muito visando a questão do desenvolvimento típico, mas são atípicos, né? Atenta-se no que é esperado para eles ou não. Eu acho que eles têm potencial, porém os estímulos são diferentes.

De modo geral, a visão que os trabalhadores da APS demonstraram aspectos correntes na atualidade, por vezes confusos e contraditórios, tanto no meio técnico/profissional quanto na sociedade: pessoas com SD podem ser consideradas "normais" ou "diferentes". Tais discursos indicaram que está havendo mudanças na tradicional concepção de que pessoas com $\mathrm{SD}$, são necessariamente pessoas deficientes, com comportamentos inadequados quando comparados aos da população em geral. Por fim, convém considerar que as crenças dos profissionais geram comportamentos que podem ser mais ou menos humanizados, incentivadores e transformadores.

Tema II: As percepções de trabalhadores da Atenção Primária à Saúde sobre a saúde de pessoas com Síndrome de Down

Os participantes de nível médio e técnico destacaram a fragilidade da saúde de pessoas com SD, conforme revelado no DSC 5.

DSC 5: Elas necessitam de um cuidado maior, de uma atenção maior, porque são pessoas um pouco mais sensíveis.... Ela é mais frágil do que uma criança normal, às vezes, ela tem vários problemas de pneumonia, de internações por este caso. Então, são mais frágeis para algumas doenças, aspiram mais alguns cuidados. É mais fragilizado não em questão de pegar um resfriado, ter dor de barriga, essas situações, não! Eu acho também que de uma certa forma, eles apresentam alguma coisa a mais, principalmente a obesidade. Eu acho que o fator mais agravante deles é a obesidade, porque eles têm aquela tendência de se alimentar, de querer comer mais. Eles têm mais limitações na formação da arcada dentária. Tu vês... assim... de fala também. 
Já os trabalhadores de nível superior (especialmente os especialistas não médicos) destacaram que a saúde de pessoas com SD requer cuidados especiais, enquanto os da área médica consideraram a saúde de pessoas com SD como a das que não têm a síndrome, conforme DSC 6 e DSC 7, respectivamente. Note que a visão dos profissionais de nível técnico e a dos de superior não médicos é compatível.

DSC6: São pessoas mais vulneráveis que a população em geral, eles têm uma expectativa de vida menor, tem uma incidência de patologias cardíacas muito frequentes e, por isso, um tipo de vida um pouco diferente que os padrões normais, isso torna-os mais vulneráveis. ...mais frágeis. Mais frágeis que a população em geral. O coração... tem toda uma necessidade de acompanhamento. A questão bucal tem bastante comprometimentos, eles têm diferenças, tanto na língua como na formação como um todo. A obesidade também, outro fator que preocupa. Tem bastante problemas respiratórios, problema de deglutição, muito problema motor, dificuldade dos marcos do desenvolvimento quando crianças. Então eles são sempre bem atrasados, demoram para sentar-se, demoram para falar, alguns tem muita dificuldade para falar, no decorrer da vida toda. Eu entendo que a saúde do paciente com SD requer uns cuidados específicos, existem algumas coisas, algumas situações de saúde que são mais comuns nessa população e que precisam de acompanhamento e de atendimento, tanto da parte biológica, quanto da parte cognitiva. Não entendo que a saúde deles seja comparável à de uma pessoa que não tem SD. Eu imagino que eu precise destinar um atendimento diferenciado para eles. Entretanto, claro que há vários graus de acometimentos de algumas patologias, mas a gente sabe de pessoas com SD que podem ter uma vida com bastante controle de alguns acometimentos de saúde e que podem ter uma vida com uma qualidade boa.

DSC 7: Eu acho que eles têm a saúde normal, alimentação normal, cuidado, higiene, tudo. Acho que é bem igual a qualquer outra pessoa. Levam uma vida normal. Eu acho que em tempos passados eles tinham essa vulnerabilidade, que se pode dizer assim né? As pessoas tinham menos recursos, então parecia que as pessoas teriam essas deficiências de saúde geral; hoje como tem a Medicina avançada, eles são considerados pessoas com saúde normal. Não é uma coisa assim específica que eu identifico por ser doente, por ter a síndrome, só como qualquer outro. Eu penso que eles são como nós, eles apresentam algumas cardiopatias, que se desde que nascem sejam acompanhadas por especialistas, não tem problema. Depois eles consultam por doenças comuns como todo mundo. Só não tem uma qualidade boa de vida se a família não buscar mesmo né? Mas tem condições de viver bem.

Note-se que houve concordância entre os profissionais de nível técnico como os de nível superior - os primeiros reconheceram que a criança com SD é mais suscetível a pneumonias e, quando maiores à obesidade. As doenças respiratórias são decorrentes de problemas comuns - imunidade prejudicada e vias áreas estreitas ou, ainda, por lesões estruturais raras (brônquio traqueal). Estudos (Watts \& Vyas, 2013), enfatizam que compreender essa gama de potenciais problemas auxilia na avaliação e gestão de pessoas com SD. Já os profissionais, considerando os avanços da medicina, referiram que, quando há problemas orgânicos, estes são passíveis de correção (Freire, 2010). No mais, os cuidados primários para adultos com SD são semelhantes ao da população geral. No entanto, autores destacam que há adição de exames de triagem para condições específicas que são mais comuns na síndrome do que na população em geral (Jensen \& Bulova, 2014).

Cabe ressaltar, que as pessoas com deficiência intelectual, sobretudo aquelas com SD, podem apresentar envelhecimento atípico, com declínio funcional acentuado (Carvalho, Ardore \& Castro, 2015) associado à multimorbidades, como Diabetes Melitus, obesidade e Hipertensão Arterial Sistêmica, alterações de personalidade, deterioração no autocuidado, declínio da capacidade cognitiva, adaptativa e de socialização, alterações afetivas súbitas, apatias, perda de vocabulários e tendência à manifestação da Doença de Alzheimer (Novell, Nadal, Smilges, Pascual \& Pujol, 2010).

Adultos com SD podem apresentar várias condições clínicas concomitantes e sobrepostas; geralmente recebem vários medicamentos e tratamentos, em particular, medicamentos psicotrópicos e, às vezes, não contam com apoio familiar e social adequados. Tal condição pode ainda ser mais complicada por prejuízos funcionais e cognitivos, que aumentam os riscos para o desenvolvimento de síndromes específicas, incluindo problemas comportamentais e nutricionais. A prevalência dessas condições aumenta após os 40 anos de vida. Essa complexidade de cuidados exige a superação das práticas tradicionais da 
medicina junto a crianças, jovens e adultos, assemelhando-se aos cuidados geralmente exigidos pelos idosos (Glasson, Dye \& Bittles, 2014).

\title{
Tema III: $O$ cuidado integral de pessoas com Síndrome de Down
}

Os DSC 8 e DSC 9 são relativos ao cuidado integral à saúde das pessoas com SD referidos pelos trabalhadores de nível superior, sendo o DSC 8 produzido por residentes multiprofissionais e o DSC 9 por trabalhadores das equipes mínimas das unidades de saúde. Note-se nos discursos o reconhecimento de que ainda há fragmentação na Rede de Cuidado conforme prevista no SUS, e a importância da interdisciplinaridade e/ou comunicação entre os profissionais de um serviço (APS) e outros para se alcançar a integralidade do cuidado. Há, também, no DSC 9 referências aos limites da inclusão social de pessoas com SD.

\begin{abstract}
DSC 8: No geral, eu acho que a SD precisa de uma atenção mais do que especial, porque ainda o SUS oferece um cuidado muito fragmentado na SD; aí a importância do cuidado integral. ...eles precisariam ser mais bem acompanhados... por uma da equipe multiprofissional. Eu percebo muita dificuldade das famílias, a questão de recursos... a gente vê que eles têm essas dificuldades. A experiência aqui na unidade é boa, porque a gente tem a oportunidade de fazer um atendimento de forma integral, ver a pessoa como um todo, ver a pessoa em sua totalidade e não só como uma patologia, como fazem habitualmente os especialistas, só veem uma parte do paciente e acham que o paciente é "departamentalizado". Aqui a Unidade é uma ESF e a gente tem a residência profissional em atenção primária, tem nutricionista, tem psicólogo, tem assistente social, tem terapeuta ocupacional e estes profissionais têm o conhecimento e a gente da Unidade quer incluir essas pessoas (pessoas com SD), eles [os residentes] estão ou vão fazer bastante trabalhos de grupo, incluir também essas pessoas nessas atividades. A gente está tentando fazer um trabalho voltado para parte promoção e prevenção, com grupo de convivência que engloba todas as idades. A questão multiprofissional e interdisciplinar... porque, muitas vezes, oque que acontece: a gente da Unidade fica muito restrita ao médico, ao enfermeiro e ao técnico de enfermagem, a equipe mínima, e ele [pessoa com SD] é um usuário que precisa ter um outro olhar, ele precisa da Fisioterapia, de Fonoaudiólogo, da Terapia Ocupacional, ele precisa da Psicologia. Muitas vezes, ela pode não entender o que a psicóloga está colocando, mas o familiar, a mãe, o pai, o tio, enfim... quem for o responsável precisa ser apoiado para que possa também estar auxiliando no processo de desenvolvimento e, até de envelhecimento. O cuidado integral é importante, sobretudo quando tem vulnerabilidades sociais, elas ficam em condições menos favoráveis para diferentes coisas. E a vulnerabilidade, na parte social, que eles têm mesmo. A gente trabalha buscando o cuidado integral do sujeito, independentemente de ser síndrome ou não. A SD é só uma característica a mais na vida dela. Então, nesse sentido, a gente sempre trabalha com o sujeito, independente se é paciente com algum tipo de deficiência, enfim... se trabalha na lógica da integralidade.
\end{abstract}

DSC 9: Eu não me vejo, hoje, preparada adequadamente para fazer essa abordagem integral, nem ao paciente com $S D$, nem a família. A gente tenta fazer o possível, mas eu acredito que em algum ponto possa ser melhorado. E como lidar com isso né? Que atividades dar para essa pessoa? Porque a gente fala na inclusão social, mas a gente também não sabe. As escolas, na verdade, não estão preparadas, a gente como profissional de saúde muitas vezes não está preparada e aí eu não sei inclui essa pessoa. Nas capacitações, é falado, é discutido as coisas relacionadas a esse cuidado mais integral, mas eu acho que ainda é bem incipiente, não se fala muito, não tem muita capacitação, não tem uma linha de cuidado ou alguma coisa mais integral para esses usuários. Eu acho que é bem incipiente essa temática. A gente não tem uma coisa voltada para essa população, mas a gente quando vem, a gente tenta ter um olhar diferenciado para essa pessoa, falta um pouco desse olhar. Eu vejo assim o cuidado de saúde aqui, muito focado na procura que a família tem, muito pontual. Então é quando aquela família tem uma demanda para resolver daquele paciente, traz o paciente e a gente resolve aquilo, mas não mantem, não tem um cuidado longitudinal com aquela família. Não tem uma continuidade ou às vezes não sabe, não se comunica com outros profissionais que possam estar atendendo aquele paciente. Essa comunicação é muito falha.

Torna-se relevante discutir a importância de os cursos de formação em saúde, valorizar a formação interdisciplinar e intersetorial, a fim de amenizar dificuldades e favorecer a qualidade, o acesso aos serviços de atenção à saúde e à educação necessária dessa população. Nobrega-Therrien, Almeida \& Silva (2008) e Saviani (2009), indicam que a formação em saúde tende a ser tecnicista e especializada, apesar de as Diretrizes Curriculares Nacionais para Formação em Saúde (Brasil, 2001) 
independentemente da especificidade de cada curso a formação na área da saúde sustenta-se em competências e habilidades gerais as quais devem regular a formação generalista, critica e reflexiva dos egressos. Nideck \& Queiroz (2015) afirmam que as instituições de ensino não conseguem ainda responderem às exigências da sociedade e do mundo do trabalho (em transição), por isso é equivocado considerar que um profissional recém-formado chega totalmente apto; segundo os autores é preciso dar continuidade ao processo de formação após a graduação.

Conforme explicitado nos DSC 8 e DSC 9 ficou evidente a necessidade de equipes multidisciplinares, no mínimo, com abordagem interdisciplinar para realizar assistência integral à saúde - seja de pessoas com ou sem SD. A mudança do modelo de atenção (uni para multiprofissional) requer mudança de paradigma. Requer a adoção de uma concepção ampliada de saúde (Brasil, 1997). Assim, as diretrizes da integralidade, qualidade, equidade e participação social devem ser concretizadas em ações coletivas centradas no desenvolvimento humano e na promoção da saúde, capazes de produzir saúde para além do marco individualista e assistencialista.

O projeto é ambicioso e pode parecer utópico quando se avalia que o perfil formativo da maior parte dos profissionais de saúde ainda está muito distante desse horizonte e que muitos obstáculos serão encontrados no percurso (Mângia \& Lancman, 2008). Por fim, pode-se dizer que as características orgânicas, cognitivas e sociais de uma pessoa com SD impõem à pessoa acometida, à família e, também, aos profissionais de saúde desafios adicionais quanto ao cuidado (Luiz, 2008; Vello et al., 2014; Silva et al., 2017).

A fragmentação do cuidado em relação as pessoas com SD foi percebida neste estudo. Cecílio \& Merhy (2003) defendem que a linha de cuidado deve atravessar inúmeros serviços de saúde, visa um grande pacto realizado entre todos os atores envolvidos, sendo o usuário o elemento estruturante de todo processo de produção da saúde. A adoção dessa estratégia objetiva evitar a fragmentação dos serviços de saúde no acompanhamento de usuários, por meio de fluxos organizados, garantindo à população a integralidade, acesso ao sistema e às ações de saúde segundo suas necessidades, de modo que o usuário seja realmente o ponto central do SUS, em lugar dos serviços e procedimentos.

A situação encontrada, em síntese, contribui para uma menor resolutividade das ações em saúde, com possível agravamento das condições de saúde dessas pessoas, piorando sua qualidade de vida. Mais do que um sistema, diversos autores (Cecílio \& Merhy, 2003; Cecílio, 2001; Campos \& Domitti, 2007) defendem que se deve pensar em uma rede móvel, assimétrica e incompleta de serviços que operem distintas tecnologias de saúde e que sejam acessados de forma desigual pelas diferentes pessoas ou agrupamentos que deles necessitam.

Frente ao exposto, a organização de linhas de cuidado deve se pautar na produção de um pacto na rede assistencial, integrando todos os recursos disponíveis por meio de fluxos capazes de garantir o acesso seguro às tecnologias necessárias à assistência. A linha de cuidado é fruto desse pacto realizado entre todos os atores que controlam os serviços e recursos assistenciais (Franco \& Magalhães Júnior, 2004).

Por fim, pode-se dizer que são muitas pesquisas que destacam a importância do acompanhamento multiprofissional de pessoas com SD visando diminuir a prevalência de complicações (Roizen \& Patterson, 2003; Moeller et al., 2006; Pikora et al., 2014). Têm-se disponível, na atualidade, protocolos de atendimento clínico em vários países, por exemplo o da Academia Americana de Pediatria (2005). No Brasil, em 2013, foi lançado, pelo Ministério da Saúde, o primeiro documento especificamente voltado às pessoas com SD - as Diretrizes de Atenção à Pessoa com Síndrome de Down (Brasil, 2013); um produto que pode ser consumido por trabalhadores da saúde de todos os níveis de atenção. O objetivo desta diretriz é oferecer orientações às equipes multiprofissionais para o cuidado à saúde da pessoa com SD, nos diferentes pontos de atenção da rede de serviço ao longo do seu ciclo vital. Refere cuidado do nascimento à velhice de pessoas com SD. 
Tema IV: O (re)conhecimento acerca da AMRPI e a prospecção do cuidado à saúde de pessoas com Síndrome de Down a partir dela

Os DSC 10 e DSC 11 revelam as opiniões de trabalhadores de nível superior e o DSC 12 dos trabalhadores de nível técnico sobre a AMRPI.

\begin{abstract}
DSC 10: Eu acho que esse tipo de avaliação é pouco visto, principalmente no momento da graduação. Essa questão da gerontologia, do cuidado com o idoso, acho que está surgindo de um tempo para cá, mas...acho que ela não refletiu ainda nas matrizes curriculares dos cursos da área da saúde. Então a avaliação multidimensional da pessoa idosa...? A gente conhece o que tem na Caderneta da Pessoa Idosa. Eu tenho pouca informação. Seria uma avaliação em todos os níveis, né? Ver ela [pessoa idosa] perante a sociedade, a família, o trabalho dela... tudo mais, né? Eu tive contato com a Avaliação Multidimensional na disciplina de geriatria, durante a graduação. Eu sei que ela leva em conta vários aspectos do envelhecimento. Ela leva em conta tanto a parte biológica, cognitiva, social, de dependência, qualidade de vida. É interessante. Ela demanda do profissional certo conhecimento e demanda, sobretudo, sua disponibilidade para se envolver com isso.
\end{abstract}

DSC 11: Olha, A avaliação que a gente faz junto aos idosos especificamente, é uma avaliação holística: onde eles estão morando, o que eles estão fazendo e o que eles podem fazer. Eu acho importante isso. A gente tenta fazer essa avaliação; eu acho que é uma questão que a gente precisa trabalhar ainda melhor. É um tema muito interessante, complexo, no qual eu acho que tem que estar toda a área de saúde envolvida nesse tema. Temos informações, mas lamentavelmente as tabelas [os protocolos] indicados para se usar aqui no Brasil são bastante complexas e, muitas vezes, não há oportunidade de mais profissionais se envolverem para se fazer uma avaliação correta. A gente utilizou algumas vezes a Avaliação Multidimensional na implantação da Caderneta da Pessoa Idosa. A gente teve capacitação da Secretaria da Saúde, da Política da Saúde da Pessoa Idosa; toda equipe foi capacitada com aqueles dados relativos a saúde mental deles, aos riscos de quedas, vários itens e os agentes comunitários de saúde, na verdade aplicaram com os idosos nas casas deles. Então, a gente conseguiu fazer um mapeamento desses idosos.

DSC 12: São aquelas questões que tem na Carteirinha do Idoso só que com o nome diferente [risos]. Inclusive a gente está trabalhando com essa carteirinha para alguns pacientes e fazendo as anotações da medicação, fazendo avaliação do grau de dificuldade dentro de sua própria residência.... É bem interessante saber que existe uma política, né? Eu já tenho conhecimento porque eu fiz o Curso de Cuidados com Idosos, então, eu já vi essa (AMRPI), uma planilha que tu fazes os testes, daí tu vai dando uma nota para o idoso e vai ver se ele tem a possibilidade ou não de ficar sozinho. A gente teve capacitações sobre esse assunto pela Secretaria de Saúde. É novo e é bem extenso; não é só tu fazer uma ou duas perguntas, tu tens todo um trabalho de pesar, de medir, de ver a perspectiva social, econômica.... É mesmo ver o paciente como um todo.

Estudos afirmam que a avaliação multidimensional é o esforço sistemático de organizar o grande número de informações advindas do processo investigativo, com a intenção de manejar apropriadamente a vida de um idoso em seus complexos e interativos problemas. Define-se como um método de diagnóstico, frequentemente interdisciplinar, preocupado em detectar problemas psicossociais e funcionais, com a finalidade de incrementar um plano de acompanhamento a longo prazo, assertivo e efetivo (Netto \& Brito, 2001; Sirena, 2002; Moraes, 2012).

Note-se que os dados deste estudo revelaram conhecimento dos trabalhadores quanto ao fato de a AMRPI ser um procedimento de avaliação que visa compreender o processo global/integral da saúde e que tem como principal objetivo a definição do diagnóstico multidimensional e do plano de cuidados (Moraes, 2012). Revelaram que estão devidamente orientados, pelos responsáveis da Política Municipal do Idoso, a usarem a AMRPI para implantarem e/ou implementarem o uso da Caderneta do Idoso, tanto, que consideraram a AMRPI como importante para a adequada prestação de cuidados à população idosa. Foi indicado que a AMRPI inclui, além da avaliação das funções física e mental, a identificação do suporte sociofamiliar, os aspectos ambientais e a necessidade de tratamento de doenças crônicas. Foi dito ainda que a AMRPI é longa e trabalhosa de ser aplicada, mas não foi, em momento algum, indicada a inviabilidade de seu uso. 
Os participantes também apresentaram em seus discursos seu entendimento que a AMRPI como uma proposta positiva para a avaliação e acompanhamento da pessoa com SD na APS, conforme observado nos DSC a seguir.

DSC 13: Então, eu tive contato com a Avaliação Multidimensional na disciplina de geriatria, durante a graduação. Eu sei que ela leva em conta vários aspectos do envelhecimento. E tu me trouxestes essa reflexão agora, de que a Avaliação Multidimensional, talvez na SD ela até seria uma "baita" ferramenta, além dela ser muito útil e interessante para o idoso, na SD talvez ela seja um plus, algo a mais, né? Sabemos que o idoso requer um olhar bem mais específico, porque ele já é considerado com vulnerabilidade; ele já não consegue desempenhar aquelas funções do dia a dia como ele desempenhava há um tempo né. Daí você pensa numa pessoa com SD idosa, dupla vulnerabilidade, e que não é bem trabalhada. Não é difundido; tem aí essa uma lacuna, uma população esquecida.

O DSC 13 revela a condição de trabalhadores de ensino superior que não tinham atentado para a possibilidade de adotar a AMRPI como orientadora para avaliar e acompanhar pessoas com SD. Na medida em que houve questionamento sobre, afirmou-se a potencialidade de seu uso no cotidiano do cuidado de uma pessoa com SD na APS, ou seja, considerou-se possível usá-la como norteadora para buscar uma avaliação abrangente (porque multidimensional) para compreensão das diversas comorbidades, dos déficits funcionais e/ou cognitivos experimentados por adultos e idosos com SD, que tendem a não mais serem acompanhados nos serviços especializados - sejam ambulatórios ou escolas - tampouco pela avaliação médica tradicional. Destaca-se, aqui, que mais pesquisas são necessárias para examinar o impacto de tal avaliação nos serviços e resultados entre adultos com SD.

Segue-se o DSC 14 que revelou, por parte de trabalhadores de nível superior, a conveniência de a AMRPI ser usada junto a pessoas com SD na APS de forma bem assertiva.

DSC 14: Sem dúvida há uma carência. Uma carência teórica e prática. É.... eu penso que não tem nada específico de idoso, de pessoa com SD com mais idade. Eu acho que seria bem válido se tivesse. Eu acho que ainda falta bastante coisa para gente aprender sobre a saúde das pessoas especiais. Tudo vai somar para o nosso conhecimento, mesmo que os casos sejam poucos. Seria bem importante que tivéssemos maior subsídio para trabalhar com pessoas com idade mais avançada que têm SD. Eu acho que cursos de capacitação, sempre são bem-vindos, educação permanente, questão de materiais, de recursos materiais mesmo, uma estrutura melhor também, estrutura física, um piso adequado, as paredes, enfim toda a estrutura física da unidade. A questão de recursos humanos, que a gente tem uma equipe desfalcada, precisamos de mais profissionais e que bom se a gente conseguisse além desses profissionais que estão faltando, aumentar esses outros profissionais que também fazem parte, porque é o que a gente precisa. Eu acho que esse modelo de médico e enfermagem, ele já está ultrapassado há muito tempo. E a gente precisa assim avançar e enxergar as outras profissões também como parte do cuidado em saúde, não numa coisa isolada, mas dentro da equipe, trabalhando com a equipe também, sendo parte mesmo, integrante dessa equipe. A gente devia entender muito mais as limitações que eles podem ter que são diferentes dos outros.

No DSC 15 apresenta-se as opiniões de trabalhadores de nível médio e técnico, especialmente de ACS, a respeito da necessidade de procedimentos novos para contribuir com a avaliação e acompanhamento da saúde de pessoas com SD.

DSC 15: Seria interessante. Eu acho que a gente tem que ter mais capacitações, porque hoje é de um jeito, amanhã é de outro. Eu também acho que a gente ter mais reuniões de rede, porque se a rede está funcionando, fica bem mais fácil para nós aqui, né? Nós somos a ESF, a porta de entrada. Eu, inclusive, acho que a gente devia ter capacitações em todas as síndromes, com SD, com paralisia cerebral, porque, às vezes, a gente pensa que tudo é igual e não é! Teria que ter capacitação, muito bem-feita, bem elaborada para a gente saber lidar com o paciente e com o familiar. Saber como lidar com eles. Quando tem deficiência mental, tem uma mentalidade de criança, mas num corpo que está envelhecendo e eu não sei como lidar com isso. Quanto mais conhecimento a gente tiver da síndrome em si, quanto mais a gente tiver atualizados sobre os tratamentos, as maneiras que existem de avaliar e amenizar a limitação de cada um, melhor. É interessante, é importante para qualquer profissional de saúde, não só para nós, agentes, eu como agente de saúde comunitário de saúde. 
Neste estudo, também foi possível constatar que os trabalhadores, independentemente do nível de formação, apresentaram em seus discursos intenções de transformar suas práticas e, assim, a realidade do atendimento a pessoas com SD na APS, conforme os DSC 16 e DSC 17 de trabalhadores com nível superior e com nível médio/técnico, respectivamente.

DSC 16: Eu acho que a gente deveria ter um instrumento; a primeira coisa capacitar e a segunda, é acolher mais esses pacientes, trazer mais para dentro da unidade. Eu acho que é bem importante sim ter alguma coisa para a gente se embasar e ter essa relação com eles e com o familiar. É bem importante. A gente não tem suporte para evidenciar mais essas pessoas. Acho interessante a criação de um protocolo, nessa linha de fazer essa avaliação multi como é a do idoso. Muito interessante mesmo. Eé isso que você vai fazer? Até para ter uma avaliação, para depois tu ter um parâmetro para comparar no futuro. Muito importante. Eu acho importante um instrumento que nos guie, porque, às vezes, têm perguntas, questionamentos que a gente não sabe como fazer. Daí quando tu trazes essa problemática para dentro da unidade, com entrevista, com esse trabalho que tu estás desenvolvendo, eu acho que isso é bem interessante para dar uma mexida com a gente. Faz pensar, puxa vida, a SD, já faz tempo que eu não penso sobre isso [risos], como está o meu olhar... Então eu acho que seria muito legal a gente ter mais contato com formas de cuidado, com o cuidar deles de uma forma melhor. Fiquei feliz que tu trouxeste essa questão para nós e eu espero que o teu trabalho renda muitos frutos e mexa com muitas cabeças por aí [risos]. E, de repente, talvez isso possa alavancar alguma capacitação ou alguma coisa a mais em relação à Rede, porque eu entendo que, assim como eu estou com essa dificuldade, pode ter muitos colegas que estão com ela, né? Esta é uma forma da gente mexer um pouquinho com a gestão, alertando para ela estar qualificando os trabalhadores para mudar o olhar sobre isso. Sim eu vejo necessidade, eu acho que podia ser algum tipo de projeto. Eu acho que, em primeiro lugar, teríamos que ver com as agentes comunitárias. Pedir para elas fazerem a busca ativa e daí, em equipe, pensar um projeto; pensar nas ações que a gente poderia fazer para esse tipo de população.

DSC 17: Eu sinto necessidade de algo que me oriente como proceder. Qualquer aprendizado é válido. A gente, meio como o que dizem tu não tens formação, tu não és enfermeira, tu não és médica, não és técnica -, sim! Mas a gente tem escuta ... como o médico, a enfermeira. A gente vai aprendendo, o ouvido fica apurado. O conhecimento, a gente adquire e pode usar dentro de casa, na família que está ao lado, onde a gente está atendendo, ou seja, em todo relacionamento interpessoal. A gente vai ter que aprender como é que eles vão poder envelhecer. Eu gostaria de ter mais base nisso aí, acho que essa tua pesquisa vai fazer, se tu descobrir como é que os que já estão mais velhinhos, como é que eles chegaram lá, como eles se comportaram, a gente vai poder aprender. Acho que deveria começar do zero assim, sabe, capacitações, dinâmicas, ir até as instituições, como eu te disse a gente não tem aquele trabalho específico com a SD.

Note-se o alerta que o desenvolvimento deste estudo produziu junto aos trabalhadores da APS. Constatação de que atualmente é raro atender as pessoas com SD nos serviços de saúde; identificando-se inúmeras barreiras presentes no acesso as unidades de saúde por essa população - das arquitetônicas, passando pelas de conhecimento técnico e de gestão dos serviços. Ficou evidente a conveniência de os trabalhadores desse nível de atenção à saúde conhecerem a legislação que ampara as pessoas com SD e dessa forma incentivar, enquanto saúde, a inclusão social (Lopes, Soares \& Bousch, 2014). A APS também tem responsabilidades, como parte integrante de uma rede de cuidados às pessoas com deficiência. Reconhece-se que a APS ainda enfrenta grandes desafios para sua própria consolidação enquanto ordenadora e coordenadora da rede do cuidado (Brasil, 2006). É, pois, mister que trabalhadores e gestores do SUS se empenhem para a prestação de um serviço de qualidade e efetivo para toda a população, incluindo, as pessoas com SD. A associação das políticas de saúde precisa acontecer para que, de fato, cumpram-se os princípios da universalidade, equidade e integralidade do SUS.

A legislação para pessoas com deficiência, por exemplo, apesar de ser bem elaborada e ampla, necessita ser mais bem implementada e fiscalizada, para que se possa construir ações de prevenção de deficiência e facilitação do acesso à saúde e à educação, entre outros direitos de cidadania. A Política Nacional de Saúde e da Pessoa Portadora de Deficiência (Brasil, 2008) tem como diretrizes - promoção da qualidade de vida, assistência integral, prevenção das deficiências, ampliação e fortalecimento dos mecanismos de informação, organização dos serviços de atenção as pessoas com deficiência e capacitação de recursos humanos. 
A Política enfatiza que seu propósito é a inclusão plena desse sujeito, protegendo sua saúde e prevenindo agravos. Ainda, traz a importância das equipes de Atenção Primária e dos agentes comunitários na identificação da situação de saúde e seus determinantes, bem como uma maior possibilidade de capilarização do cuidado às pessoas, conhecedores que são do seu modo de viver e de como seu contexto histórico-cultural pode influenciar no processo saúde-doença, já que desenvolvem ações dentro de um território adscrito e pelo vínculo que possuem com aquela comunidade, família e indivíduo (Brasil, 2008).

No entanto, a assistência ainda se apresenta fragilizada e desarticulada, inserindo-se marginalmente nos sistema de saúde (Girondi \& Santos, 2011), apesar de que se tem prevista a Rede do Sistema Público organizada em serviços de Atenção Básica, Atenção Especializada em Reabilitação (Auditiva, Física, Intelectual, Visual, Ostomia e em Múltiplas Deficiências), Atenção Hospitalar e de Urgência e Emergência, que, articulados entre si, ou seja, com devida regulação, garante o acesso a quaisquer pontos da Rede e, assim, a integralidade do cuidado (Brasil, 2012).

Além do explicitado acima, destaca-se o Plano "Viver Sem Limite", (Brasil, 2011), que tem como objetivo, promover por meio da integração e da articulação de políticas, programas e ações, o exercício pleno e equitativo dos direitos da pessoa com deficiência. O Plano "Viver sem Limite" apresenta quatro grandes frentes de atuação: Acesso à Educação, Inclusão Social, Acessibilidade e Atenção à Saúde. Em cada uma delas, ações específicas são planejadas e organizadas para atingir a um número cada vez maior de pessoas com deficiência, nas diferentes esferas sociais (Brasil, 2011).

Por fim, ainda pertinente a esses aspectos, tem-se a Lei Brasileira de Inclusão da Pessoa com Deficiência - Estatuto da Pessoa com Deficiência - (Brasil, 2015) que determina que é dever do Estado, da família, da comunidade escolar e da sociedade assegurar à pessoa com deficiência, seu direito à saúde e à educação, dentre outros, ofertando uma rede de serviços articulados, com atuação intersetorial nos diferentes níveis de complexidade respeitando os territórios e as normas do SUS, garantindo acesso universal e igualitário.

\section{Conclusão}

De acordo com o objetivo proposto constatou-se que a compreensão dos trabalhadores da APS sobre a saúde das pessoas com SD é incipiente: as concepções dos trabalhadores sobre essas pessoas estão atreladas além das diferenças físicas, as diferenças cognitivas e de comportamento dessas pessoas. Quanto às percepções sobre a saúde, destacaram-se as condições clínicas concomitantes e sobrepostas - Diabetes Melitus, obesidade e Hipertensão Arterial Sistêmica, alterações de personalidade, deterioração no autocuidado, declínio da capacidade cognitiva, adaptativa e de socialização, alterações afetivas súbitas, apatias, perda de vocabulários e tendência à manifestação da Doença de Alzheimer.

Os trabalhadores não consideravam a AMRPI para assistir as pessoas com SD e, quando a (re)conheceram, identificaram-na como uma proposta positiva para a avaliação e acompanhamento dessa população na APS. Os trabalhadores também reconheceram que estão pouco preparados para receber, avaliar e acompanhar, em suas unidades, usuários com SD.

Consideraram que é necessária formação - por meio da Educação Permanente - acerca da temática discutida neste estudo. Também indicaram pertinente aproximar a AMRPI como procedimento de cuidado a pessoas com SD, visto que ela se apresenta como efetivação do cuidado integral à saúde. Reconheceram o desafio, a necessidade de reflexão sobre as práticas atuais e sobre novas possibilidades de atenção no nível da APS.

Acompanhar as mudanças instituídas pelo atual contexto populacional brasileiro - acelerado envelhecimento - é dever de todos, bem como o planejamento de ações que valorizem o cuidado mais humanizado, com efetiva comunicação entre os trabalhadores das equipes e pontos da rede de assistência.

Pode-se dizer que o desenvolvimento deste estudo funcionou, para os trabalhadores de saúde, como um despertar para o aprimoramento de seus conhecimentos acerca das possibilidades de atenção à saúde de pessoas com SD. Vale ressaltar que os achados do presente estudo se limitam a uma realidade específica. Assim, sugere-se a realização de investigações desta 
natureza, em novos cenários, no intuito de se alcançar uma melhor compreensão das possibilidades de cuidado à saúde de pessoas com SD em seus contextos/comunidades.

\section{Referências}

American Academy of Pediatrics Task Force on Sudden Infant Death Syndrome. (2005). The changing concept of sudden infant death syndrome: diagnostic coding shifts, controversies regarding the sleeping environment, and new variables to consider in reducing risk. Pediatrics, 116 (5), $1245-1255$.

Aranha, M. S. F. (2001). Paradigmas da Relação da Sociedade com Pessoas com Deficiência. Brasília: Revista do Ministério Público do Trabalho, ano XI, 21,160- 173 .

Bonamigo, A. W. \& Rodrigues, P. V. P. (2009). Aspectos históricos e conceituais do programa saúde da família ao NASF. In: $17^{\circ}$ Congresso Brasileiro de Fonoaudiologia, Rev Soc Bras Fonoaudiol. Supl Esp, 2382.

Brasil. (1997). Ministério da Saúde. Secretaria de Assistência à Saúde. Coordenação de Saúde da Comunidade. Saúde da família: uma estratégia para reorientação do modelo assistencial. Brasília: Ministério da Saúde.

Brasil. (2006). Ministério da Saúde. Portaria $\mathrm{n}^{\circ} 2.528$ de 19 de outubro de 2006. Aprova a Política Nacional de Saúde da Pessoa Idosa. http://bvsms.saude.gov.br/bvs/saudelegis/gm/2006 prt2528_19_10_2006.html

Brasil. (2006). Ministério da Saúde. Secretária de Atenção à Saúde. Departamento de Atenção Básica. Política Nacional de Atenção Básica. Brasília: Ministério da Saúde.

Brasil. (2008). Ministério da Educação. Secretaria de Educação Especial. Política Nacional de Educação Especial na Perspectiva da Educação Inclusiva. Brasília. http://portal.mec.gov.br//arquivos/pdf/politicaeducespecial.pdf.

Brasil. (2008). Ministério da Educação. Secretaria de Atenção à Saúde. Política Nacional de Saúde da Pessoa Portadora de Deficiência. Série Legislações em Saúde. 1 ed. Editora Ministério da Saúde: Brasília (DF).

Brasil. (2008). Ministério da Saúde. Portaria no 154 de 24 de janeiro de 2008. Cria os Núcleos de Apoio à Saúde da Família - NASF. Brasília: Ministério da Saúde.

Brasil. (2011). Decreto 7612 de 17 de novembro de 2011. Institui o Plano Nacional de Direitos da Pessoa com Deficiência - Plano Viver Sem Limites. http://www.planalto.gov.br/ccivil_03/_ato2011-2014/2011/decreto/d7612.htm.

Brasil. (2012). Ministério da Saúde. Conselho Nacional de Saúde. Resolução No 466, de 12 de dezembro de 2012. Ministério da Saúde.

Brasil. (2013). Ministério da Saúde. Secretaria de Atenção à Saúde. Departamento de Ações Programáticas Estratégicas. Diretrizes de atenção à pessoa com Síndrome de Down / Ministério da Saúde, Secretaria de Atenção à Saúde, Departamento de Ações Programáticas Estratégicas. Ministério da Saúde.

Brasil. (2015). Lei no 13. 146, de 6 de julho de 2015. Institui a Lei Brasileira de Inclusão de Pessoas com Deficiência (Estatuto da Pessoa com Deficiência). Brasília.

Campos, G. W. S. \& Domitti, A. C. (2007). Apoio matricial e equipe de referência: uma metodologia para gestão do trabalho interdisciplinar em saúde. Cadernos de Saúde Pública, v. 23 (2), 339-407.

Canesqui, A. M. \& Spinelli, M. A. S. (2006). Saúde da Família do estado do Mato Grosso, Brasil: perfis e julgamentos dos médicos e enfermeiros. Cad. Saúde Pública, 22 (9), 1881-1892.

Carvalho, C. L., Ardore, M. \& Castro, L. R. (2015). Cuidadores Familiares e o Envelhecimento da Pessoa com Deficiência Intelectual: Implicações na prestação de cuidados. Revista Kairós Gerontologia, 18 (3), 333-352.

Casarin, S. (2001). Os vínculos familiares e a identidade da pessoa com Síndrome de Down. Mestrado em Psicologia Clínica/PUC.

Cecílio, L. C. O. (2001). As necessidades de saúde como conceito estruturante na luta pela integralidade e equidade na atenção à saúde. In: Pinheiro, R. \& Mattos, R. A (org.). Os sentidos da integralidade na atenção e no cuidado à saúde. IMS-UERJ-ABRASCO.

Cecílio, L. C. O. \& Merhy, E. E. (2003). A integralidade do cuidado como eixo da gestão hospitalar. In: Pinheiro, R. \& Mattos, R. A. Construção da integralidade: cotidiano, saberes e práticas em saúde. IMS/ Abrasco.

Duncan, B. B., Chor, D., Aquino, E. M. L., Bensenor, I. M., Mill, J. G., Schimidt, M. I., Lotufo, P. A., Vigo, A. \& Barreto, S. M. (2012). Doenças Crônicas Não Transmissíveis no Brasil: prioridade para enfrentamento e investigação. Rev Saúde Pública, 46 (1), $126-134$.

Franco, T. B. \& Magalhães Júnior, H. M. (2004). Integralidade na assistência à saúde: a organização das linhas do cuidado. In: MERHY, E. E. et al. (orgs). O trabalho em saúde: olhando e experienciando o SUS no cotidiano. HUCITEC.

Freire, R. C. L., Melo, S. F. \& Hazin, I. (2014). Aspectos neurodesenvolvimentais e relacionais do bebê com Síndrome de Down. Av. Psicol. Latinoam, Bogotá, 32 (2), 247-259.

Giaccini, V., Tonial, A. \& Mota, H. B. (2013). Aspectos de linguagem e motricidade oral observados em crianças atendidas em um setor de estimulação precoce. Distúrbios da comunicação, 25 (2), 253-265.

Giordani, J. M. A., Cezar, P. K., Campos, G., Kretzmman, F. G. \& Kocourek, S. (2015). Características dos profissionais de saúde da família no atendimento de violência contra crianças e adolescentes. Rev. Enferm. UFSM, 5 (2), 316-326. 
Girondi, J. B.R. \& Santos, S. M. A. (2011). Deficiência física em idosos e acessibilidade na atenção básica em saúde: revisão integrativa da literatura. Rev. gaúcha enferm. 31 (2), 378-384.

Glasson, E. J., Dye, D. E. \& Bittles, A. H. (2014). Os desafios triplos associados com comorbidades relacionadas à idade na síndrome de Down. $J$ Intellect Disabil Res., 58, 393-398. 10.1111 / jir.12026

Goulart, F. A. A. (2012). Doenças crônicas não transmissíveis: estratégias de controle e desafios e para os sistemas de saúde. Brasília: Ministério da Saúde.

Huber, J., Peres, V. C., Santos, T. J., Beltrão, L. F., Baumont, A. C., Cañedo, A. D., Schaan, B. D. \& Pellanda, L. C. (2010). Cardiopatias congênitas em um serviço de referência: Evolução Clínica e Doenças Associadas. Arquivo Brasileiro de Cardiologia, $94 \quad$ (3), $333-338$. <http://www.scielo.br/pdf/abc/v94n3/09.pdf>

Lefevre, F. \& Lefevre, A. M. C. (2000). Os novos instrumentos no contexto da pesquisa qualitativa. In: Lefèvre, F., Lefèvre, A. M. C. \& Teixeira, J. J. V. organizadores. O discurso do sujeito coletivo: uma nova abordagem metodológica em pesquisa qualitativa. Caxias do Sul: EDUCS, 11-35.

Lopes, M. J. M., Soares, J. S. F. \& Bohusch, G. (2014). Usuários portadores de deficiência: questões para a atenção primária de saúde. Revista Baiana de Enfermagem. Salvador, 28 (1). Disponível em: < https://portalseer.ufba.br/index.php/enfermagem/article/view/8496/8703>. Acesso em 03/11/2019.

Luiz, F. M. R., Bortoli, P. S., Floria-Santos, M. \& Nascimento, L. C. (2008). A inclusão da criança com Síndrome de Down na rede regular de ensino: desafios e possibilidades. Rev. bras. educ. espec, 14 (3), 497-508. Disponível em: < http://dx.doi.org/10.1590/S1413-65382008000300011.

Mângia, E. F. \& Lancman, S. (2008). Núcleos de apoio à saúde da família: integralidade e trabalho em equipe multiprofissional. Rev Ter Ocup. 19 (2), i-i. https://doi.org/10.11606/issn.2238-6149.v19i2pi-i

Marsiglia, R. M. G. (2011). Perfil dos trabalhadores da Atenção Básica em Saúde no município de São Paulo: região norte e central da cidade. Saúde Soc. 20 (4), 900-911.

Mattos, B. M. \& Bellani, F. (2010). A importância da estimulação precoce em bebês portadores de síndrome de down: revisão de literatura. Revista brasileira de terapia e saúde, Curitiba, 1 (1), 51-53.

Mendes, E. V. (2012). O cuidado das condições crônicas na atenção primária à saúde: o imperativo da consolidação de estratégia da saúde da família. O cuidado das condições crônicas na atenção primária à saúde: o imperativo da consolidação da estratégia da saúde da família. Brasília: Organização PanAmericana da Saúde.

Minayo, M. C. S. (2014). O desafio do conhecimento: pesquisa qualitativa em saúde. Hucitec.

Moeller, M. P., Eiten, L., White, K. \& Shister, L. (2006). Strategies for Educating Physicians about Newborn Hearing Screening. Journal of the Academy of Rehabilitative Audiology, 39, 11-32.

Moraes, E. N. (2012). Atenção à saúde do Idoso: Aspectos Conceituais. Organização Pan-Americana da Saúde.

Neideck, R. L. P. \& Queiroz, P. P. (2015). Perspectivas para o ensino de na saúde: do apagão educacional à política de educação permanente. Trabalho Educação e Saúde. Rio de Janeiro, 13 (1), 159-179.

Netto, M. P. \& Brito, F. C. (2001). Urgências em Geriatria. Ed. Atheneu.

Nobrega-Therrien, S. M., Almeida, M. I. \& Silva, M. G. C. (2008). Ensino de Enfermagem no Ceará de 1942- 1956: memória que projeta o futuro. Revista Brasileira de Enfermagem, 61 (1), 125-130. http://www.scielo.br/scielo.php?script==sci_arttext\&pid=S0034-71672008000100021.

Novell, R., Nadal, M., Smilges, A., Pascual, J. \& Pujol, J. (2010). Informe Seneca: Envejecimiento y discapacidad intelectual en Cataluña. Desc. Física. Recuperado em 15 maio, 2016, de: http://sid.usal.es/24881/8-4-2

Pastore, R., Rosa, L. D. \& Homem, I. D. (2008). Relações de gênero e poder entre trabalhadores da área da saúde. In: Seminário Internacional Fazendo gênero-Corpo, Violência e Poder, 8. 〈http://www.fazendogenero.ufsc.br/8/sts/ST25/Pastore-Rosa-Homem_25.pdf>.

Pikora, T. J., Bourke, J., Bathgate, K., Foley, K.R., Lennox, N. \& Leonard, H. (2014). Health conditions and their impact among adolescents and young adults with Down syndrome. PLoS One, 9 (5), e96868. https://doi.org/10.1371/journal.pone.0096868

Pueschel, S. (2003). Causas da síndrome de Down. In: Pueschel, S. (Org.). Síndrome de Down: guia para pais e educadores. Papirus.

Rachidi, N. \& Lopes, C. (2008). Mental retardation and associated neurological dysfunctions in Down syndrome. A consequence of dysregulation in critical chromosome 21 genes and associated molecular pathways. European Journal of Paediatric Neurology, 12, 168-182. http://www.sciencedirect.com/science/article/pii/S1090379807001535

Resende, M. C., Azevedo, E. G. S., Lourenço, L. R., Faria, L. S., Alves, N. F., Farina, N. P., Silva, N. C. \& Oliveira, S. L. (2011). Saúde mental e ansiedade em agentes comunitários que atuam em saúde da família em Uberlândia (MG, Brasil). Ciênc Saúde Coletiva, 16 (4), 2115-2122.

Ribeiro, S. F. R. \& Martins, S. T. F. (2011). Sofrimento psíquico do trabalhador da saúde da família na organização do trabalho. Psicol Estud, 16 (2), $241-250$. DOI: http://dx.doi.org/10.1590/S1413-73722011000200007

Ribeiro, L. M. A., Jacob, C. M. A., Pastorino, A. C., Kim, C. A. E., Fomin, A. B. F. \& Castro, A. P. B. M. (2003). Avaliação dos fatores associados a infecções recorrentes e / ou graves em pacientes com síndrome de Down. Jornal de Pediatria. Rio de Janeiro, 79, (2), 141-148. www.scielo.br/pdf/jped/v79n2/v79n2a09

Roizen, N. J. \& Patterson, D. (2003). Down's syndrome. Lancet, 361 (9365), 1281-1289.

Saviani, D. (2009). Escola e Democracia. (41a ed.), Autores Associados. 
Research, Society and Development, v. 10, n. 9, e21510917901, 2021

(CC BY 4.0) | ISSN 2525-3409 | DOI: http://dx.doi.org/10.33448/rsd-v10i9.17901

Silva, C. G., Sena, L. B., Rolim, I. L. T. P., Sousa, S. M. A. \& Sardinha, A. H. L. (2017). Cuidados de enfermagem a pacientes com condições crônicas de saúde: uma revisão integrativa. Rev Pesqui Cuid Fundam, 9 (2), 599-605.

Sirena, S. A. (2002). Avaliação multidimensional do idoso: uma abordagem em atenção primária à saúde. 99 p. Tese (doutorado) - Pontifícia Universidade Católica do Rio Grande do Sul.

Valcarenghi, R. V., Lourenço, L. F. L., Siewert, J. S. \& Alvarez, A. M. (2015). Produção científica da Enfermagem sobre promoção de saúde, condição crônica e envelhecimento. Rev Bras Enferm, 68 (4), 705-712.

Vello, L. S., Popim, R. C., Carazzai, E. M. \& Pereira, M. A. O. (2014). Saúde do idoso: percepções relacionadas ao atendimento. Escola Anna Nery Revista de Enfermagem, 18 (2), 330-335.

Watts, R. \& Vyas, H. (2013). An overview of respiratory problems in children with Down's syndrome. Archives of Disease in Childhood, 98 (10), $812-817$.

Wermelinger, M., Machado, M. H., Tavares, M. F. L., Oliveira, E. S., Moyses, N. N. \& Ferraz, W. (2010). A força de trabalho do setor de saúde no Brasil: focalizando a feminilização. Revista Divulgação em Saúde para Debate, 45, 54-70.

Werneck, C. (2006). Ensaios pedagógicos. Brasília. Ministério da Educação, Secretaria de Educação Especial. 146p. III Seminário Nacional de Formação de Gestores e Educadores - Educação Inclusiva: direito à diversidade. http://dominiopublico.gov.br/download/texto/me4607.pdf.

Wiseman, F. K., Al-Janabi, T., Hardy, J., Karmiloff-Smith, A., Nizetic, D., Tybulewicz, V. L. J., Fisher, E. M. C. \& Strydom, A. (2015). A genetic cause of Alzheimer disease: Mechanistic insights from Down syndrome. Nature Reviews Neuroscience, 16 (9), 564-574. 10.1038/nrn3983 